\title{
Anomalous Cosmic Ray Composition from $\mathrm{ACE}$
}

\author{
R. A. Leske \\ California Institute of Technology, Pasadena, CA 91125 USA
}

\begin{abstract}
During solar quiet periods, the Solar Isotope Spectrometer (SIS) on the Advanced Composition Explorer (ACE) measures the composition and energy spectra of anomalous cosmic rays (ACRs) with energies $>8 \mathrm{MeV} /$ nucleon in interplanetary space at $1 \mathrm{AU}$. In particular, the spectra of individual isotopes for the ACR elements $\mathrm{N}, \mathrm{O}$, and $\mathrm{Ne}$ are studied with SIS. Intensity enhancements are found in low energy ${ }^{18} \mathrm{O}$ and ${ }^{22} \mathrm{Ne}$, with relative abundances of ${ }^{18} \mathrm{O} /{ }^{16} \mathrm{O} \sim 0.002$ and ${ }^{22} \mathrm{Ne} /{ }^{20} \mathrm{Ne} \sim 0.1$. The neon abundance ratio appears more similar to that found in the solar wind than in meteorites and is far below that determined for the galactic cosmic ray (GCR) source, indicating that GCRs contain material from sources other than just the local interstellar medium.
\end{abstract}

\section{INTRODUCTION}

In the 25 years since the discovery of unexpected intensity enhancements in low energy quiet time helium, nitrogen, and oxygen spectra [1-3], considerable progress has been made in understanding the nature of these so-called "anomalous" cosmic rays (ACRs). Early models [4,5] of ACR origins have held up remarkably well. In the accepted picture, ACRs originate from neutral atoms in the interstellar medium (ISM) that are swept into the heliosphere, ionized by solar UV or charge exchange with the solar wind, convected into the outer heliosphere by the solar wind as "pickup" ions, and accelerated to energies of $\sim 1$ to $>50 \mathrm{MeV} /$ nucleon at the solar wind termination shock. Nearly all aspects of this model have now been experimentally verified. Additional elements with high first ionization potential (which are primarily neutral in the ISM), such as $\mathrm{H}, \mathrm{Ne}$, and $\mathrm{Ar}$, have been found to have an ACR component (see, e.g., [6] and references therein); interstellar pickup ions have been directly observed [7] and their composition measured [8]; and low energy ACRs have been shown to be predominantly singly ionized (e.g., [9], and references therein).

Although other elements have been found with small enhancements at lower energies [10,11], which may originate as adsorbed, neutralized, and desorbed solar wind on dust grains [12] or grain destruction products [13], the bulk of the ACRs provide a direct sample of the neutral interstellar medium. As such, studies of the

CP516, $26^{\text {th }}$ International Cosmic Ray Conference, edited by B. L. Dingus, et al. (C) 2000 American Institute of Physics 1-56396-939-4/00/ $\$ 17.00$ 
isotopic composition of this material can shed light on the overall nucleosynthetic history of this part of the Galaxy since the formation of the solar system some 4.5 billion years ago and on the nature of galactic cosmic rays (GCRs), which may also be an accelerated sample of the present day interstellar medium [14].

The Solar Isotope Spectrometer (SIS) on the Advanced Composition Explorer (ACE) is able to significantly improve upon existing ACR isotope measurements $[15,16]$, owing to its large collecting power and good mass resolution [17]. Since the launch of ACE in August of 1997 under solar minimum conditions, solar modulation of ACRs and GCRs has increased as solar maximum approaches. Figure 1 shows daily averages of the 7-21 MeV/nucleon oxygen measured by SIS during solar quiet days, compared with the count rate of the University of Chicago's Climax neutron monitor. Clearly a strong correlation exists between the intensity of higher rigidity GCRs, as indicated by the neutron monitor, and the ACRs measured by SIS, which can detect statistically significant variations on time scales as short as one day. Note that the ACR intensity has fallen by a factor of $\sim 5$ between the launch of ACE and June 1999. The SIS elemental and isotopic data reported here cover quiet time periods from the launch of ACE through late March 1998, when ACR intensities were high and relatively constant.

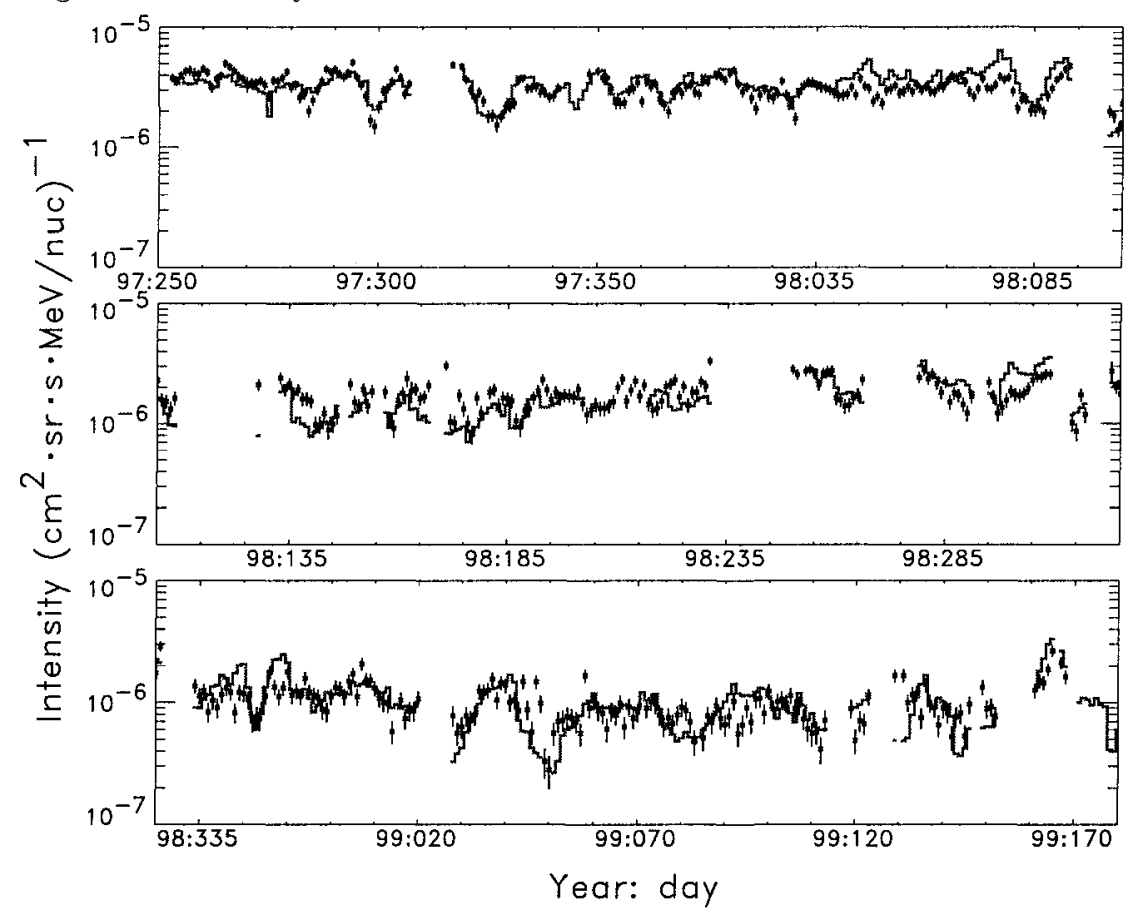

FIGURE 1. Daily average fluxes of 7-21 MeV/nucleon oxygen from SIS (data points), compared with the count rate of the Climax neutron monitor (histogram; actually, (Climax/6691.8) ${ }^{28.1}$, to fit on the same scale) during solar quiet days since the launch of ACE. 


\section{ELEMENTAL SPECTRA}

The SIS instrument consists of a pair of silicon solid-state detector telescopes and uses the $\mathrm{d} E / \mathrm{d} x$ versus residual energy technique to measure the nuclear charge, $Z$, mass, $M$, and total kinetic energy, $E$, of energetic particles which stop in the device [17]. The ACR species of interest here are measured in energy intervals which range from 6.6-83.3 MeV/nucleon for $\mathrm{N}$ to $7.8-101.8 \mathrm{MeV} /$ nucleon for Ne. Mass resolution varies from $\sim 0.15$ to $\sim 0.4 \mathrm{amu}$, depending on $E$ and $Z$.

Elemental spectra of selected species from SIS are shown in Figure 2. To better illustrate the overall shape of the spectra, higher energy data from the Cosmic Ray Isotope Spectrometer (CRIS) on ACE [18] and lower energy data from the LowEnergy Matrix Telescope (LEMT) on Wind [10] are also included in the figure. The time period of the Wind data overlaps that of the SIS measurements, but extends from November 1994 to April 1998. Most of the quiet time periods used in the Wind analysis, however, were in 1996 and early 1997, and the ACR fluxes during this time were rather similar to those in late 1997 and early 1998 [10]. The agreement between measurements from all three instruments represented in Figure 2 is generally remarkably good.
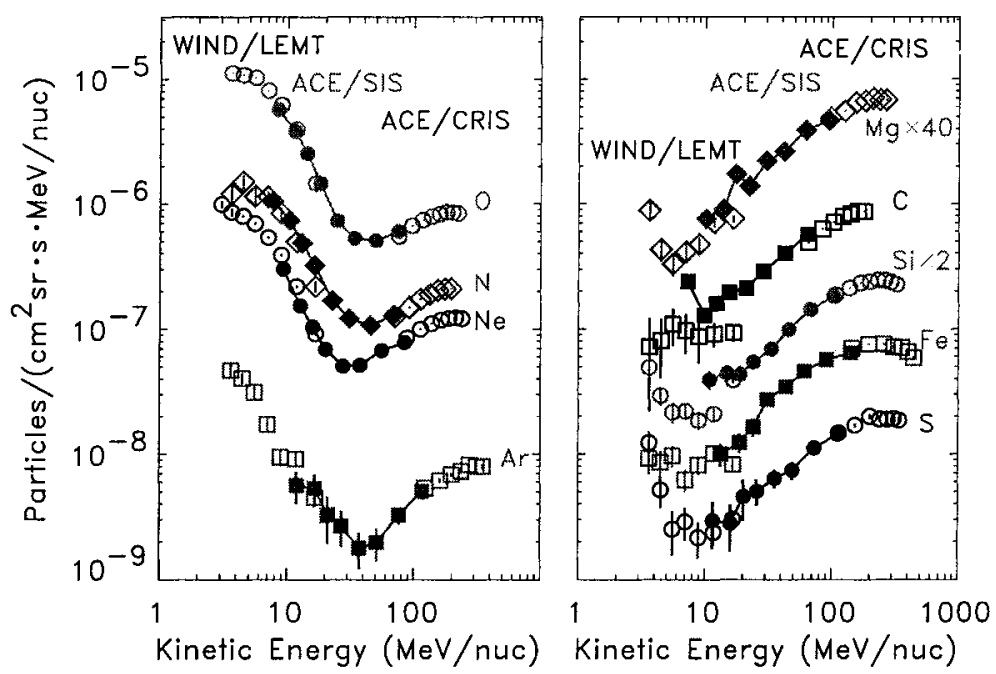

FIGURE 2. Elemental spectra from the SIS (filled symbols) and CRIS (high energy open symbols) instruments on ACE from 8/27/97 to 3/23/98 [19]. Lower energy trends (open symbols) are illustrated by data from the LEMT instrument on Wind [10] covering a similar time period. Statistical uncertainties are shown, which are often much smaller than the plotted symbol.

Aside from a few discrepant points in these preliminary SIS data at low energies, where background events are difficult to eliminate, and small differences between CRIS and SIS and between SIS and LEMT for N and O, the biggest difference is 
between LEMT and SIS for C. Although the lowest energy SIS point may include some background from residual solar material or from other misidentified elements (although this is unlikely to account for the full factor of 2 increase above the trend extrapolated from higher energies), the higher energy data should be free of such problems. In the Wind instrument, a large amount of background has been subtracted from the $\mathrm{C}$ data [10], as is reflected in the large size of the $\mathrm{C}$ uncertainties compared with those of species with much lower intensities. Given the large size of these corrections, a factor of 2 difference from SIS is probably not unreasonable, but the question of just how much $\mathrm{ACR} C$ is present at $1 \mathrm{AU}$ under strict quiet time conditions appears to be unresolved. Data from SAMPEX $[20,21]$ indicate that some of this $\mathrm{C}$ is not highly ionized and may be ACRs, though sources other than interstellar neutrals are possible [13].

SIS does not observe particles at energies low enough to detect the low energy enhancements recently reported for $\mathrm{Mg}, \mathrm{Si}, \mathrm{S}$, and perhaps $\mathrm{Fe}$ at $1 \mathrm{AU}$ [10] and in the outer heliosphere [11]. (Although no enhancement for Fe is claimed in [10], some appears to be present compared with the trend extrapolated from SIS measurements). The ACR Ar enhancement is clearly observed by SIS, however the statistical accuracy is too low to subdivide the data and obtain meaningful isotopic measurements at this time. Therefore, this report is limited to ACR isotopes for $\mathrm{N}, \mathrm{O}$, and $\mathrm{Ne}$. Additional details on these measurements are provided by Leske et al. [22].

\section{ISOTOPIC ANALYSIS}

Mass distributions of $\mathrm{N}, \mathrm{O}$, and $\mathrm{Ne}$ in two energy intervals each are shown in Figure 3. Consistency among multiple determinations of $Z$ and $M$ was required in order to reject events that involved chance coincidences or underwent nuclear interactions in the instrument. Also, the measured trajectory of each particle was used to identify and reject particles which could have exited through the sides of the instrument without stopping.

The composition variation with energy evident in Figure 3 reflects the fact that the isotopic abundances are different for ACRs and GCRs. Above $\sim 50$ $\mathrm{MeV} /$ nucleon, where GCRs dominate, most of the observed ${ }^{15} \mathrm{~N}$ and ${ }^{18} \mathrm{O}$ is produced by cosmic ray spallation during transport through the Galaxy and is not directly representative of either the GCR source or the local ISM abundances, while most of the observed ${ }^{22} \mathrm{Ne}$ originates in the cosmic ray source. Below $\sim 30$ $\mathrm{MeV} /$ nucleon, ACRs are the dominant contributor to the quiet time fluxes. Since, unlike GCRs, the ACRs have passed through a negligible amount of material, no secondary spallation products should be present.

The energy dependence of the isotopic composition is more clearly illustrated by the spectra of individual ACR isotopes shown in Figure 4. Because of the likelihood of background from the neighboring peaks, we report only an upper limit on the lowest energy ${ }^{18} \mathrm{O}$ value, while a $20 \%$ systematic uncertainty has been 

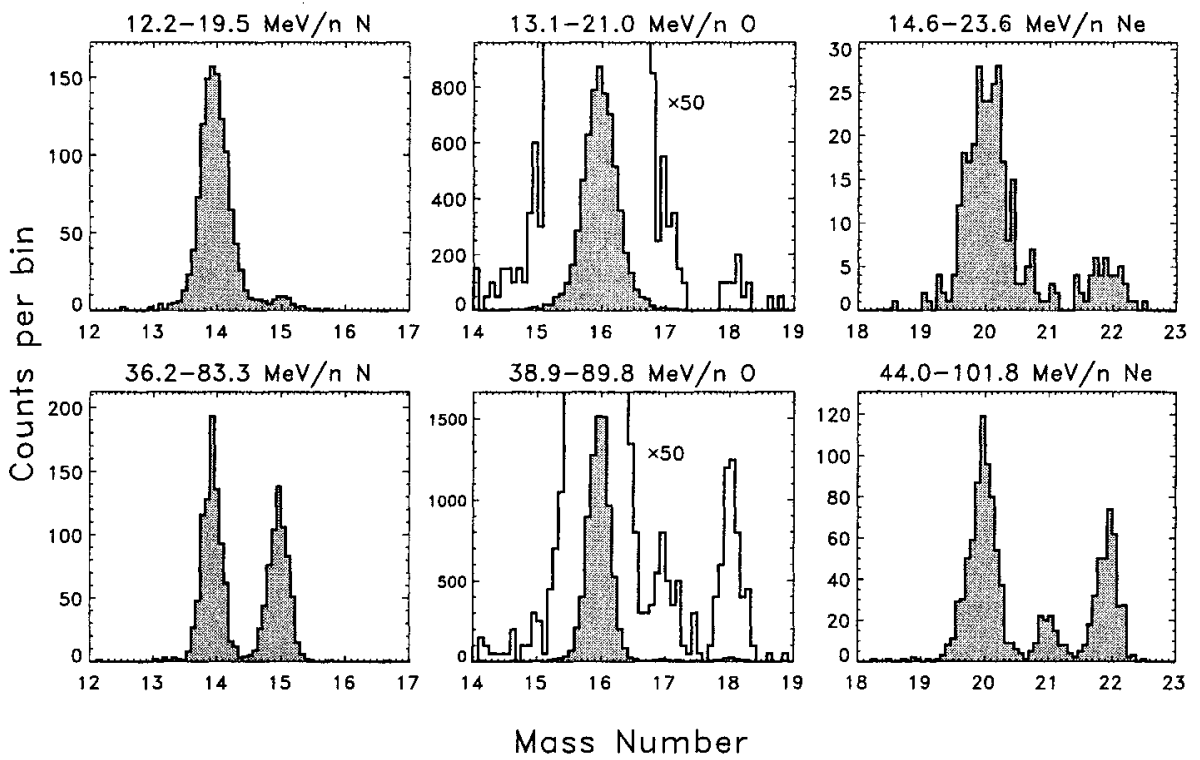

FIGURE 3. $\mathrm{N}, \mathrm{O}$, and Ne isotope distributions from SIS at the indicated energies. A factor of 50 expansion of the $\mathrm{O}$ histograms is used to better illustrate the ${ }^{17} \mathrm{O}$ and ${ }^{18} \mathrm{O}$ peaks.

added in quadrature to the statistical uncertainties in the ${ }^{22} \mathrm{Ne}$ values below 15 $\mathrm{MeV} /$ nucleon.

Although ${ }^{14} \mathrm{~N}$ shows the typical ACR enhancement at low energies, ${ }^{15} \mathrm{~N}$ has no statistically significant enhancement. We take the slope of the ${ }^{15} \mathrm{~N}$ spectrum to be characteristic of that of GCRs in this charge and energy interval after solar modulation. Scaling this spectrum to pass through the high energy ${ }^{14} \mathrm{~N}$ points and subtracting it from the ${ }^{14} \mathrm{~N}$ spectrum yields the ACR ${ }^{14} \mathrm{~N}$ spectrum shown by the filled squares in Figure 4. An $\mathrm{ACR}{ }^{15} \mathrm{~N} /{ }^{14} \mathrm{~N}$ ratio of 0.0037 , as is found in solar system material [23], would result in the ACR ${ }^{15} \mathrm{~N}$ spectrum shown by the scaled ${ }^{14} \mathrm{~N}$ curve at the bottom of the figure. Note that a ${ }^{15} \mathrm{~N}$ abundance in ACRs consistent with the solar value would not be detectable by SIS at these energies.

On the other hand, the analogous figure for $\mathrm{O}$ isotopes (middle panel of Figure 4) shows a clear low-energy increase in the ${ }^{18} \mathrm{O}$ flux. Furthermore, the observed intensity of ACR ${ }^{18} \mathrm{O}$ after subtracting the GCR background (filled diamonds) is in good agreement with the $\mathrm{ACR}{ }^{16} \mathrm{O}$ curve scaled by the solar system ${ }^{18} \mathrm{O} /{ }^{16} \mathrm{O}$ ratio of 0.002 [23]. Thus, ${ }^{18} \mathrm{O}$ is present in ACRs, with an ${ }^{18} \mathrm{O} /{ }^{16} \mathrm{O}$ value consistent with solar system abundances.

No enhancement of ${ }^{21} \mathrm{Ne}$ is evident (right panel of Figure 4), and none is expected if the $\mathrm{ACR}{ }^{21} \mathrm{Ne} /{ }^{20} \mathrm{Ne}$ ratio is similar to that in the solar system $(0.0024 ;[23])$. However, ${ }^{22} \mathrm{Ne}$ shows a significant low energy ACR enhancement. Curves $(a)$ through (c) in Figure 4 show the expected ${ }^{22} \mathrm{Ne}$ spectrum, if the ACR ${ }^{22} \mathrm{Ne} /{ }^{20} \mathrm{Ne}$ ratio is 


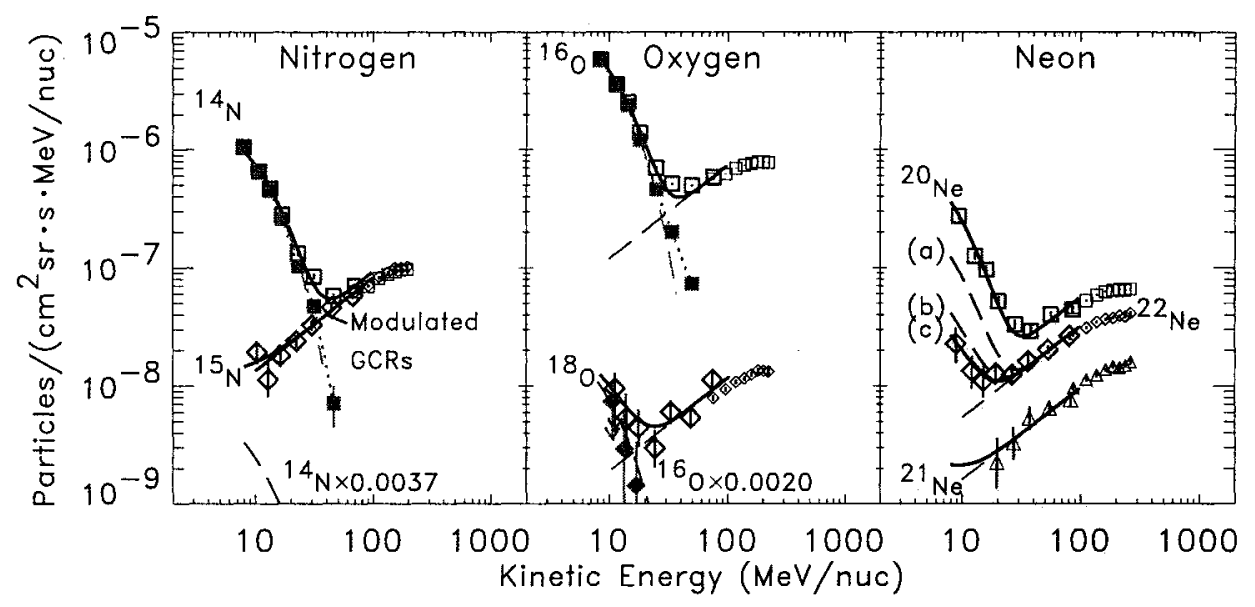

FIGURE 4. SIS quiet time isotopic energy spectra from $8 / 97-3 / 98$ for $\mathrm{N}$ (left), O (center), and Ne (right). Open symbols are measured values, while filled symbols are obtained by subtracting the GCR background. The locations of the ${ }^{14} \mathrm{~N}$ and ${ }^{16} \mathrm{O}$ ACR spectra scaled by the solar system ${ }^{15} \mathrm{~N} /{ }^{14} \mathrm{~N}$ and ${ }^{18} \mathrm{O} /{ }^{16} \mathrm{O}$ values, respectively, are also shown to indicate where the ACR ${ }^{15} \mathrm{~N}$ and ${ }^{18} \mathrm{O}$ spectra may be expected. Lettered curves show the expected ACR ${ }^{22} \mathrm{Ne}$ spectrum if the $\mathrm{ACR}{ }^{22} \mathrm{Ne} /{ }^{20} \mathrm{Ne}$ ratio is that found in the GCR source $(a)$, meteoritic Neon-A $(b)$, or the solar wind $(c)$. Smaller symbols above $\sim 80 \mathrm{MeV} /$ nucleon are data from CRIS (N. E. Yanasak, private communication).

the same as that in $(a)$ the GCR source, $(b)$ the meteoritic component Neon-A, or (c) the solar wind. The observed $\mathrm{ACR}{ }^{22} \mathrm{Ne}$ intensity is clearly well below that of the GCR source, where the ${ }^{22} \mathrm{Ne} /{ }^{20} \mathrm{Ne}$ ratio is calculated to be between 0.322 [24] and 0.448 [25], depending on the GCR transport model and fragmentation cross sections used in interpreting the measurements. The ${ }^{22} \mathrm{Ne}$ intensity also appears low compared to what it would be if the ${ }^{22} \mathrm{Ne} /{ }^{20} \mathrm{Ne}$ ACR ratio were similar to that of the meteoritic component Neon- A, with ${ }^{22} \mathrm{Ne} /{ }^{20} \mathrm{Ne}=0.122$ [26]. It agrees well, however, with curve $(c)$, which assuines that the ${ }^{22} \mathrm{Ne} /{ }^{20} \mathrm{Ne}$ ACR ratio is the same value as that found in the solar wind, 0.073 [27].

\section{RESULTS AND DISCUSSION}

Although SIS does not observe energies low enough to measure the ACR ${ }^{15} \mathrm{~N}$ abundance, it would be of great interest to determine this value, since astronomical observations suggest that it may not agree with solar system values. The standard ${ }^{15} \mathrm{~N} /{ }^{14} \mathrm{~N}$ ratio in the solar system [23] is $\sim 50 \%$ higher than is observed by radio astronomers in molecular clouds at a similar distance from the galactic center (e.g., see review by Wilson and Rood, [28]). The reason for this is unclear. Possible causes include galactic chemical evolution since the formation of the solar system 
or the effect of radial diffusion of stars on galactic abundance gradients [29].

The ${ }^{18} \mathrm{O} /{ }^{16} \mathrm{O}$ ratio found in ACRs is similar (within a factor of $\sim 1.65$ ) to that found in the solar system. It does appear to be less than that observed closer to the galactic center, as shown in Figure 5, but in agreement with observations nearby in the Galaxy.

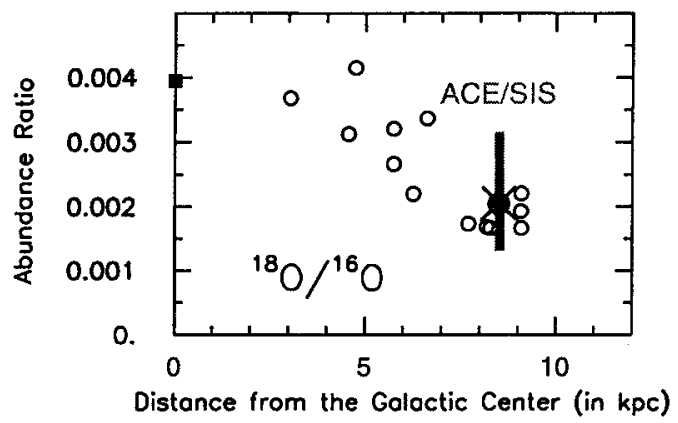

FIGURE 5. Measured ${ }^{18} \mathrm{O} /{ }^{16} \mathrm{O}$ ratio as a function of distance from the galactic center from Wilson and Rood [28]. Data from molecular cloud measurements (open circles), the galactic center (square), and the solar system (filled circle, [23]) are shown, compared with the ACE/SIS measurement (cross and vertical bar) from ACRs.

The isotopic composition of $\mathrm{Ne}$, as a noble gas, cannot be measured remotely with molecular transition lines, as $\mathrm{N}$ and $\mathrm{O}$ can. The ${ }^{22} \mathrm{Ne} /{ }^{20} \mathrm{Ne}$ ratio measured in a variety of samples of matter varies considerably, as shown in Figure 6. NeonA, which is found in carbonaceous chondrite meteorites, was adopted as the solar system standard Ne composition by Cameron [26]. A component less enriched in ${ }^{22} \mathrm{Ne}$, Neon-B, is found in lunar and meteoritic materials and is thought to be implanted solar wind (see e.g. [30]), which has a similar isotopic composition (e.g. [27]). Measurements of solar energetic particles (SEPs) from both SIS and ULEIS on $\mathrm{ACE}$ have revealed that the observed ${ }^{22} \mathrm{Ne} /{ }^{20} \mathrm{Ne}$ ratio is highly variable from event to event $[31,32]$, apparently due to rigidity-dependent mass fractionation in the acceleration and/or transport of these particles, from a source which presumably is compositionally similar to the solar wind. All of these solar system samples (with the exception of unusual meteoritic components due to cosmic ray spallation or nuclear reactions [30]) are depleted in ${ }^{22} \mathrm{Ne}$, or enriched in ${ }^{20} \mathrm{Ne}$, relative to the GCR source.

Final numbers require a more careful evaluation of systematic effects and background, especially at the lowest energies. However, it is clear that the isotopic composition of ACR Ne is similar to that of solar system material, and unlike that of the source of GCR Ne. The idea that the GCR source Ne composition may be typical of the interstellar medium, and that the composition of the solar system is peculiar [33] is therefore not supported. As deduced from earlier measurements $[15,16]$, the Ne results indicate that GCRs are not just an accelerated sample of 


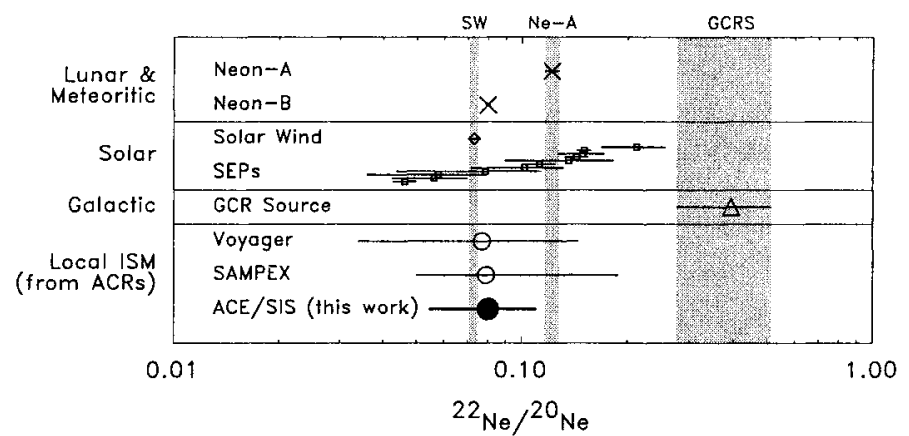

FIGURE 6. The measured ${ }^{22} \mathrm{Ne} /{ }^{20} \mathrm{Ne}$ ratio in various samples of matter, including lunar and meteoritic Ne-A [26] and Ne-B [30], solar wind [27] and SEPs [31], the GCR source [24,25], and ACRs from Voyager [16], SAMPEX [34], and ACE/SIS (this work). Unlike the lunar and meteoritic values, which are averaged over many samples, the ${ }^{22} \mathrm{Ne} /{ }^{20} \mathrm{Ne}$ ratio in 11 individual SEP events is displayed.

ISM material (although this may be the source of the bulk of low energy GCRs [14]), but apparently include contributions from other sources especially rich in ${ }^{22} \mathrm{Ne}$, such as the stellar winds of Wolf-Rayet stars [35].

\section{ACKNOWLEDGMENTS}

This research was supported by NASA at the California Institute of Technology (under grant NAG5-6912), the Jet Propulsion Laboratory, and the Goddard Space Flight Center. We thank the large group of individuals who contributed to the development of the SIS instrument [17] and the SIS science collaborators R. A. Mewaldt, E. R. Christian, C. M. S. Cohen, A. C. Cummings, P. L. Slocum, E. C. Stone, T. T. von Rosenvinge, and M. E. Wiedenbeck. Climax neutron monitor data were obtained from the web, courtesy of the University of Chicago, National Science Foundation grant ATM-9613963.

\section{REFERENCES}

1. Garcia-Munoz, M., Mason, G. M., and Simpson, J. A., ApJL 182, L81-L84 (1973).

2. Hovestadt, D., Vollmer, O., Gloeckler, G., and Fan, C. Y., Phys. Rev. Lett. 31, L650-L653 (1973).

3. McDonald, F. B., Teegarden, B. J., Trainor, J. H., and Webber, W. R., ApJL 187, L105-L108 (1974).

4. Fisk, L. A., Kozlovsky, B., and Ramaty, R., ApJL 190, L35-L37 (1974).

5. Pesses, M. E., Jokipii, J. R., and Eichler, D., ApJL 246, L85-L88 (1981).

6. Simpson, J. A., Adv. Space Res. 16, (9)135-(9)149 (1995). 
7. Möbius, E., Hovestadt, D., Klecker, B., Scholer, M., Gloeckler, G., and Ipavich, F. M., Nature 318, 426-429 (1985).

8. Geiss, J., Gloeckler, G., Mall, U., von Steiger, R., Galvin, A. B., and Ogilvie, K. W., Astron. Astrophys. 282, 924-933 (1994).

9. Klecker, B. et al., ApJL 442, L69-L72 (1995).

10. Reames, D. V., ApJ 518, 473-479 (1999).

11. Cummings, A. C., Stone, E. C., and Steenberg, C. D., Proc. 26th ICRC (Salt Lake City) 7, 531-534 (1999).

12. Fahr, H. J., Ripken, H.W., and Lay, G., Astron. Astrophys. 102, 359-370 (1981).

13. Geiss, J., Gloeckler, G., and von Steiger, R., Space Sci. Rev. 78, 43-52 (1996).

14. Meyer, J.-P., Drury, L. O'C., and Ellison, D. C., ApJ 487, 182-196 (1997).

15. Leske, R. A., Mewaldt, R. A., Cummings, A. C., Cummings, J. R., Stone, E. C., and von Rosenvinge, T. T., Space Sci. Rev. 78, 149-154 (1996).

16. Cummings, A. C., Stone, E. C., and Webber, W. R., Proc. 22nd ICRC (Dublin) 3, 362-365 (1991).

17. Stone, E. C. et al., Space Sci. Rev. 86, 357-408 (1998).

18. Stone, E. C. et al., Space Sci. Rev. 86, 285-356 (1998).

19. Christian, E. R. et al., Proc. 26th ICRC (Salt Lake City) 7, 559-560 (1999).

20. Mewaldt, R. A., Cummings, J. R., Leske, R. A., Selesnick, R. S., Stone, E. C., and von Rosenvinge, T. T., GRL 23, 617-620 (1996).

21. Oetliker, M., Klecker, B., Mason, G. M., McNab, M. C., and Blake, J. B., Proc. 25th ICRC (Durban) 2, 277-280 (1997).

22. Leske, R. A., Mewaldt, R. A., Christian, E. R., Cohen, C. M. S., Cummings, A. C., Stone, E. C., von Rosenvinge, T. T., and Wiedenbeck, M. E., Proc. 25th ICRC (Salt Lake City) 7, 539-542 (1999).

23. Anders, E. and Grevesse, N., Geochim. Cosmochim. Acta 53, 197-214 (1989).

24. Connell, J. J. and Simpson, J. A., Proc. 23rd ICRC (Calgary) 1, 559-562 (1993).

25. Lukasiak, A., Ferrando, P., McDonald, F. B., and Webber, W. R., ApJ 426, 366-372 (1994).

26. Cameron, A. G. W., in Essays in Nuclear Astrophysics, edited by C. A. Barnes, D. D. Clayton, and D. N. Schramm, Cambridge University Press, p. 23-43 (1982).

27. Geiss, J., Buehler, F., Cerruti, H., Eberhardt, P., and Filleux, Ch., Apollo 16 Prelim. Sci. Report, NASA SP-315 231, 14-1-14-10 (1972).

28. Wilson, T. L. and Rood, R. T., Annual Rev. Astron. Astrophys. 32, 191-226 (1994).

29. Wielen, R., Fuchs, B., and Dettbarn, C., Astron. Astrophys. 314, 438-447 (1996).

30. Podosek, F. A., Ann. Rev. Astron. Astrophys. 16, 293-334 (1978).

31. Leske, R. A., Mewaldt, R. A., Cohen, C. M. S., Cummings, A. C., Stone, E. C., Wiedenbeck, M. E., Christian, E. R., and von Rosenvinge, T. T., GRL 26, 26932696 (1999).

32. Dwyer, J. R., Mason, G. M., Mazur, J. E., Gold, R. E., and Krimigis, S. M., Proc. 26th ICRC (Salt Lake City) 6, 147-150 (1999).

33. Olive, K. A. and Schramm, D. N., ApJ 257, 276-282 (1982).

34. Leske, R. A., Mewaldt, R. A., Cummings, A. C., Stone, E. C., and von Rosenvinge, T. T., Proc. 25th ICRC (Durban) 2, 321-324 (1997).

35. Cassé, M. and Paul, J. A., ApJ 258, 860-863 (1982). 